\title{
1 Profiling of lysine-acetylated proteins in human urine
}

2 Weiwei Qin, Zhenhuan Du, He Huang, Youhe Gao*

3 Department of Biochemistry and Molecular Biology, Beijing Normal University,

4 Beijing, 100875, China

5 *Corresponding author:

6 Youhe Gao

7 Address: Department of Biochemistry and Molecular Biology, Beijing Normal University, Gene

8 Engineering and Biotechnology Beijing Key Laboratory, Beijing, 100875, P. R. of China

9 Email address: gaoyouhe@bnu.edu.cn

10 Tel. and Fax: +86 1058804382 


\section{Abstract.}

2 Biomarker is the measurable change associated with a physiological or

3 pathophysiological process, its nature is change. Contrast to the blood which is under

4 homeostatic controls, urine reflects changes in the body earlier and more sensitive

5 therefore is a better biomarker source. Lysine acetylation is an abundant and highly

6 regulated post-translational modification. It plays a pivotal role in modulating diverse

7 biological processes and is associated with various important diseases. Enrichment or

8 visualization of proteins with specific post-translational modifications provides a

9 method for sampling the urinary proteome and reducing sample complexity. In this

10 study, we used anti-acetyllysine antibody-based immunoaffinity enrichment combined

11 with high-resolution mass spectrometry to profile lysine-acetylated proteins in normal

12 human urine. A total of 629 acetylation sites on 315 proteins were identified,

13 including some very low-abundance proteins. This is the first proteome-wide

14 characterization of lysine acetylation proteins in normal human urine. Our dataset

15 provides a useful resource for the further discovery of the lysine acetylated proteins as

16 biomarker in urine.

17 Keywords: Urine, Post-translational modification, Lysine acetylation 


\section{$1 \quad 1$ Introduction}

2 Biomarker is the measurable change associated with a physiological or

3 pathophysiological process, its nature is change(1). In contrast to the blood, which is

4 controlled by homeostatic mechanisms, urine as waste in the body accumulates

5 changes. We suggest it is more sensitive to detect changes in the urine may be more

6 sensitive than in the plasma $(1,2)$. In addition, urine can be easily and non-invasively

7 collected in large quantities and does not undergo significant proteolytic degradation

8 compared to other biofluids (3). We established a urimem that adsorbs biological

9 molecules in the urine onto a membrane $(4,5)$. This method makes it possible to store

10 and archive urine samples economically on a large scale for long-term preservation.

11 Urine may become very important for large-scale biomarker research.

12 Post-translational modifications (PTMs) are covalent modifications of a protein (6)

13 and play a vital role in modulating several biological functions. In the past decade, the

evolution of mass spectrometry-based proteomic methods including enrichment

strategies has enabled wide-scale identification of PTMs, such as acetylation (7),

16 glycosylation (8), and phosphorylation (9). Lysine acetylation (Kac), a dynamic and

17 reversible PTM, was initially discovered in histones approximately 50 years ago (10).

18 Subsequent studies were focused on chromatin remodeling for gene transcription,

until the first non-histone protein, p53, was identified to be lysine-acetylated (11). In

2006, Kim et al. reported the first systematic analysis of lysine-acetylated proteins,

21 indicating that Kac is involved in the regulation of diverse cellular pathways beyond

DNA-templated processes. Subsequent studies detected abundant Kac in non-histone 
1 proteins in eukaryotes (such as in human, mouse, and Drosophila) $(7,12)$ and

2 prokaryotes (such as Escherichia coli, Salmonella enterica) (13, 14).

3 Lysine-acetylated proteins are involved in nearly all cellular processes and are

4 evolutionarily conserved from bacteria to mammals. These proteins are also

5 associated with important diseases such as metabolic diseases, neurodegenerative

6 disorders, and cardiovascular diseases(15-17).

7 In urine, only glycosylation and phosphorylation have been studied and applied in

8 biomarker research $(8,18)$. Compared to more than 400 forms of PTMs detected in

9 proteins(19), little is known about the PTMs of urine proteins. In this study, we used

10 immune-affinity-based acetyllysine peptide enrichment integrated with

11 high-resolution mass spectrometry to profile lysine-acetylated proteins in normal

12 human urine.

\section{Materials and methods}

2. 1. Ethical statement

15 The consent procedure and the study protocol were approved by the Institutional

16 Review Board of the Institute of Basic Medical Sciences, Chinese Academy of

17 Medical Sciences. (Project No. 007-2014). Written informed consent was obtained

18 from each subject prior to the study.

\section{2. 2. Urine sample preparation}

20 The first morning mid-stream urine was collected from 16 donors $(8$ males and 8

21 females), aged 24-40 years (average 30 and 27 years for males and females, 22 respectively), routine medical examinations were normal, and females who were in 
1 menses were excluded. Next, $20 \mathrm{ml}$ urine samples were filtered through a

2 nitrocellulose membrane, urinary proteins were adsorbed onto the membrane, and the

3 membrane was dried and stored in a vacuum bag as described previously (4). Urinary

4 proteins were eluted from the membrane by vortexing, followed by quantification by

5 the Bradford method for western blot analysis.

$6800 \mathrm{ml}$ pooled urine samples (3 males and 3 females, aged 24-30 years) were

7 centrifuged at $3500 \times g$ for $30 \mathrm{~min}$. After discarding the debris, the supernatant was

8 centrifuged at $12,000 \times g$ for $30 \mathrm{~min}$. After the removal of precipitates, urinary

9 proteins were fractionated by acetone and re-dissolved in lysis buffer (7 M urea, $2 \mathrm{M}$

10 thiourea, $120 \mathrm{mM}$ dithiothreitol, and $40 \mathrm{mM}$ Tris), and then quantified by the

11 Bradford method for immune-affinity enrichment and LC-MS/MS analysis.

\section{3. Western blot analysis}

13 Urinary proteins from each sample $(30 \mu \mathrm{g}, \mathrm{n}=16)$ were loaded on $12 \%$ SDS-PAGE

14 and transferred to a polyvinylidene difluoride (PVDF) membrane. After blocking in

15 TBST buffer (1X TBS containing 0. 1\% Tween 20) with 5\% (w/v) nonfat milk for 40

16 min at room temperature, the membranes were incubated with diluted primary

17 antibodies (dilution 1:1000) (PTM Biolabs, Chicago, IL, USA) at $4^{\circ} \mathrm{C}$ with gentle

18 shaking overnight. Next, the membrane was probed with secondary antibodies

19 (dilution 1:5000) coupled to horseradish peroxidase. Signals were revealed by

20 enhanced chemiluminescence, and the results were scanned and analyzed using an

21 Image Quant 400TM Imager (GE Healthcare, Little Chalfont, UK). Finally, the

membrane was stained with Coomassie Brilliant Blue. 


\section{2. 4. Protein digestion}

$25 \mathrm{mg}$ urinary proteins were used for digestion, the protein solution was reduced with

$310 \mathrm{mM}$ DTT for $1 \mathrm{~h}$ at $37^{\circ} \mathrm{C}$ and alkylated with $20 \mathrm{mM} \mathrm{IAA}$ for $45 \mathrm{~min}$ at room

4 temperature in the dark. For trypsin digestion, the protein sample was diluted by

5 adding $100 \mathrm{mM}$ ammonium bicarbonate to make the urea concentration less than $2 \mathrm{M}$.

6 Finally, trypsin was added at a 1:50 trypsin-to-protein mass ratio for the first digestion

7 overnight and 1:100 trypsin-to-protein mass ratio for the second 4-h digestion.

\section{5. Off-line high-pH HPLC separation}

$92 \mathrm{mg}$ tryptic peptides was then fractionated by high $\mathrm{pH}$ reverse-phase HPLC using

10 Agilent 300Extend C18 column (5 $\mu \mathrm{m}$ particles, $4.6 \mathrm{~mm} \mathrm{ID,} 250 \mathrm{~mm}$ length, Santa

11 Clara, CA, USA). Briefly, peptides were first separated over a gradient of 2-60\%

12 acetonitrile in $10 \mathrm{mM}$ ammonium bicarbonate, $\mathrm{pH} 10.0$ for 80 min into 80 fractions.

13 Next, the peptides were combined into 7 fractions and dried by vacuum

14 centrifugation.

\section{6. Enrichment of lysine-acetylated peptides}

To enrich Kac peptides, tryptic peptides dissolved in NETN buffer $(100 \mathrm{mM} \mathrm{NaCl}, 1$ mM EDTA, $50 \mathrm{mM}$ Tris-HCl, 0.5\% NP-40, $\mathrm{pH}$ 8.0) were incubated with pre-washed antibody beads (PTM Biolabs) at $4^{\circ} \mathrm{C}$ overnight with gentle shaking. The beads were washed four times with NETN buffer and twice with $\mathrm{ddH}_{2} \mathrm{O}$. The bound peptides were eluted from the beads with $0.1 \%$ TFA. The eluted fractions were combined and vacuum-dried. The resulting peptides were cleaned with C18 ZipTips (Millipore, Billerica, MA, USA) according to the manufacturer's instructions, followed by 
1 LC-MS/MS analysis.

\section{2. 7. LC-MS/MS analysis}

3 The lyophilized peptides were re-suspended in $0.1 \%$ formic acid and subjected to

4 chromatography using an EASY-nLC 1000 UPLC system (Thermo Scientific,

5 Waltham, MA, USA). The peptides were separated on a reversed-phase analytical

6 column (Acclaim PepMap RSLC, Thermo Scientific). Elution was performed over a

7 gradient of $7-35 \%$ buffer $\mathrm{B}(0.1 \%$ formic acid in $98 \% \mathrm{ACN}$; flow rate, $0.3 \mu \mathrm{L} / \mathrm{min})$

8 for 40 min. A Q Exactive ${ }^{\mathrm{TM}}$ Plus mass spectrometer (Thermo Scientific) was used to

9 analyze the fractions. The MS data were acquired using the high-sensitivity mode

10 with the following parameters: 20 data-dependent MS/MS scans per full scan, full

11 scans acquired at a resolution of 70,000 and MS/MS scans at a resolution of 17,500,

12 dynamic exclusion (exclusion duration 30 s), MS scans over a range of 350-1800

$13 \mathrm{~m} / \mathrm{z}$.

\section{8. Data processing}

The MS raw data were processed using the PEAKS Studio (version 7.5, Bioinformatics Solution Inc., Waterloo, Canada). High-quality de novo sequence tags were then used by PEAKS $\mathrm{DB}(20)$ to search the human proteome database (UniProtKB/Swiss-Prot release 2014-01-10). The fasta file contained 20120 protein sequences. A mass tolerance of $10 \mathrm{ppm}$ and $0.02 \mathrm{Da}$ was set for the precursor ions and fragment ions, respectively. Cysteine carbamidomethylation was set as fixed modification, oxidation (M), protein N-terminal and lysine acetylation as variable modifications. Trypsin allowing 3 missed cleavages was chosen as the enzyme. A 
1 decoy database was also searched to calculate the false-discovery rate (FDR) using

2 the decoy-fusion method. Result filtration parameters: peptide-spectrum matches FDR

$3<0.1 \%$; Peptide $-10 \operatorname{lgP} \geq 27.5$ (FDR 0.2\%); Protein $-10 \operatorname{lgP} \geq 28$ (FDR 0.0\%), and

4 proteins unique peptides $\geq 1$; AScore $\geq 59$ considers as confident Kac site.

\section{2. 9. Motif and secondary structure analysis}

6 Software motif-x was used to analyze the model of sequences with amino acids in

7 specific positions of acetylated lysine-13-mers (6 amino acids upstream and

8 downstream of the acetylation site) in all protein sequences (21). The IPI human

9 proteome was used as an extension database, while other parameters were set to

10 default values. The local secondary structures of lysine-acetylated proteins were

11 predicted by NetSurfP (22).

12 2. 10. Protein networks and functional analysis

13 All identified lysine-acetylated urinary proteins were subjected to network and

14 functional analyses using ingenuity pathway analysis (IPA) version 9.0

15 (http://www.ingenuity.com).

$16 \quad 3$ Results and discussion

3. 1. Western blot analysis of lysine-acetylated urinary proteins of healthy

humans

19 In order to explore the status of lysine acetylation in the urine proteome of healthy humans, western blot analysis was performed using a pan anti-acetyllysine antibody.

21 The results showed that multiple protein bands with a wide range of molecular weights were detected. In addition to histones (11-15 kDa and $26 \mathrm{kDa}$ ), many 
1 non-histone proteins with clear bands were observed (Fig 1B and Fig. S1), indicating

2 that lysine acetylation is highly abundant in human urine. Furthermore, the acetylated

3 proteins showed different patterns among different healthy individuals. This suggests

4 that lysine acetylation levels reflect various statuses of healthy people.

\section{3. 2. Profiling of lysine-acetylated urinary proteins}

6 Because lysine acetylation plays a vital role in modulating various molecular

7 processes and widely exists in the urine, we comprehensively profiled

8 lysine-acetylated proteins in the urine using immune-affinity-based acetyllysine

9 peptide enrichment integrated with LC-MS/MS.

10 We first identified 761 acetylated peptides (Peptide-Spectrum Matches FDR < $0.1 \%$ )

11 (Table S1) matched on 315 lysine-acetylated proteins (Unique peptides $\geq 1$ ) with 629

12 Kac sites (AScore $\geq 59$ ) in normal human urine (Table S2). Among these proteins, 18

13 contained five or more Kac sites, accounting for $5.7 \%$ of the total detected Kac

14 proteins with an average degree of acetylation of 2.

15 When these 315 proteins were searched in the most comprehensive urinary database

16 (www.urimarker.com/urine/) (Fig 2A), 242 were found in 1D group (proteins

17 detectable when separated by $1 \mathrm{D}$ LC) and 71 in the 2D and 3D group (2D: high-pH

18 RPLC coupled LC- MS/MS, 3D: GELFrEE/LP-IEF fraction prior to 2D), which were

19 less abundant proteins; 2 were not found in this database.

20 This is the first proteome-wide characterization of lysine acetylation proteins in

21 normal human urine. A total of 629 acetylation sites on 315 proteins were identified, 
1 high $\mathrm{pH}$ pre-fraction of unenriched peptides vastly reduced the sample complexity

2 and increased the dynamic range. For albuminuria occurring in some kidney diseases,

3 the masking effects of highly abundant proteins and ion suppression were much

4 stronger. To address this issue, a combination of two types of PTMs, such as

$5 \quad \mathrm{~N}$-glycosylated proteins and lysine-acetylated peptide enrichment, may be used.

\section{3. 3. Analysis of lysine acetylation sites}

7 To examine the relative abundance of the amino acids flanking the acetylation sites,

8 we analyzed the 761 lysine-acetylated peptides using Motif-X software. A negatively

9 charged residue, aspartate, at the +1 and +5 positions of acetyllysine was identified

10 (Fig 2B). According to previous studies of human lysine acetylation (23), aspartate

11 was found to be enriched at the $-1,-2$, and +1 positions of the lysine acetylation site.

12 To further explore the relationship between the lysine acetylation and protein

13 secondary structures, we performed structural analysis of all acetylated proteins using

14 NetSurfP. Acetylated lysine was found in alpha-helices approximately $8.8 \%$ more

15 frequently than the average lysine and in the coil approximately $5.5 \%$ less frequently

$16(\mathrm{p}<0.01)($ Fig $2 \mathrm{C})$. Thus, acetylated lysine appears to be enriched in structured

17 regions and depleted in unstructured regions, which is in concordance with previous

18 reports $(7,12)$.

\section{4. Functional analysis of lysine-acetylated proteins in urine}

Classification analysis of the lysine-acetylated proteins identified in urine was 
1 extracellular space, $18.8 \%$ in the plasma membrane, and $5.2 \%$ in the nucleus. The

2 proportion of cytoplasm proteins in the lysine acetylome was much higher than that in

3 the whole urinary proteome (17\%) (24), while the other proportions of extracellular

4 space or plasma membrane proteins were much lower than that in the whole urinary

5 proteome (38\% and 31\%) (24). The major types of lysine-acetylated proteins were

6 enzymes (enzyme $25.9 \%$, peptidase $11 \%$, kinase $2.3 \%$, phosphatase $1.9 \%$, and

7 transporters 10\%) (Fig 3B).

8 Enriched bio functions and diseases were analyzed using the IPA tool. As shown in

9 Fig $3 \mathrm{C}$, the notable bio functions of these proteins were cell-to-cell signaling and

10 interaction (87 proteins), cell death and survival (118 proteins), cellular growth and

11 proliferation (115 proteins), free radical scavenging (28 proteins), and cellular

12 movement (76 proteins). The major diseases and disorders associated with these

13 proteins were found to be endocrine system disorders (110 proteins), organismal

14 injury and abnormalities (299 proteins), dermatological diseases and conditions (73

15 proteins), metabolic disease (95 proteins), and neurological diseases (116 proteins).

16 These results provide valuable information regarding lysine acetylation in the urine

17 and may be useful in biomarker studies these diseases and disorders.

18 The main canonical pathways (Fig 3D) in which the lysine-acetylated proteins

19 participated, including LXR/RXR activation, acute phase response signaling, and

20 FXR/RXR activation, showed the same rankings in the whole urinary proteome.

21 Among these top 7 networks, three were associated with glucose metabolism, such as

22 glycolysis I, sucrose degradation V (mammalian), and gluconeogenesis I. 

aCC-BY-ND 4.0 International license.

1 Lysine acetylated proteins in the urine may reflect protein acetylation status in cells.

2 Discarding acetylated proteins may provide a way to modulate levels of lysine

3 acetylated proteins in cells. Our dataset provides a useful resource for the further

4 discovery of the lysine acetylated proteins as biomarker in urine. 


\section{Acknowledgements}

2 Funding: This work was supported by the National Key Research and Development

3 Program of China (grant number 2016YFC1306300); the National Basic Research

4 Program of China (grant number 2013CB530850); and funds from Beijing Normal

5 University (grant numbers 11100704, 10300-310421102).

6

7 


\section{References:}

2 1. Gao Y. Urine-an untapped goldmine for biomarker discovery? Sci China Life Sci. 3 2013;56(12):1145-6.

4 2. Li M, Zhao M, Gao Y. Changes of proteins induced by anticoagulants can be more sensitively 5 detected in urine than in plasma. Sci China Life Sci. 2014;57:649-54.

6 3. Ste' phane D, Anne P, Benjamin B, Harald M. Urine in Clinical Proteomics. Mol Cell Proteomics. $7 \quad 2008 ; 7: 1850-62$.

8 4. Jia L, Liu X, L L, al e. Urimem, a membrane that can store urinary proteins simply and 9 economically, makes the large-scale storage of clinical samples possible. Sci China Life Sci. $10 \quad 2014 ; 57: 336-9$.

11 5. Zhang F, Cheng X, Yuan Y, Wu J, Gao Y. Urinary microRNA can be concentrated, dried on 12 membranes and stored at room temperature in vacuum bags. PeerJ. 2015;3:e1082.

13 6. Mann M, Jensen ON. Proteomic analysis of post-translational modifications. Nature 14 Biotechnology 2003; 21:7.

15 7. Kim SC, Sprung R, Chen Y, Xu Y, Ball H, Pei J, et al. Substrate and functional diversity of lysine 16 acetylation revealed by a proteomics survey. Molecular cell. 2006;23(4):607-18. 8. Wang L, Li F, Sun W, Wu S, Wang X, Zhang L, et al. Concanavalin A-captured glycoproteins in healthy human urine. Molecular \& cellular proteomics : MCP. 2006;5(3):560-2.

19 9. Olsen JV, Blagoev B, Gnad F, Macek B, Kumar C, Mortensen P, et al. Global, in vivo, and site-specific phosphorylation dynamics in signaling networks. Cell. 2006;127(3):635-48.

10. Vidali G, Gershey EL, Allfrey VG. Chemical Studies of Histone Acetylation. The Distribution of EN-acetyllysine in Calf Thymus Histones. J Biol Chem. 1968;243:6361-6.

11. Gu W, Roeder GR. Activation of p53 Sequence-Specific DNA Binding by Acetylation of the p53 C-Terminal Domain. Cellular signalling. 1997;90:595-606. targets protein complexes and co-regulates major cellular functions. Science. 2009;325(5942):834-40. evolutionarily conserved modification in Escherichia coli. Molecular \& cellular proteomics : MCP. 2009;8(2):215-25. coordinates carbon source utilization and metabolic flux. Science. 2010;327(5968):1004-7.

15. Menzies KJ, Zhang H, Katsyuba E, Auwerx J. Protein acetylation in metabolism - metabolites and cofactors. Nature reviews Endocrinology. 2016;12(1):43-60.

16. Voelter-Mahlknecht S. Epigenetic associations in relation to cardiovascular prevention and therapeutics. Clinical epigenetics. 2016;8:4. acetylation modifiers in vascular remodelling: new targets for therapy in cardiovascular disease. European heart journal. 2009;30(3):266-77. on the protein level approach for the human urinary proteome: touching phosphorylation in urine. 
1 20. Zhang J, Xin L, Shan B, Chen W, Xie M, Yuen D, et al. PEAKS DB: de novo sequencing assisted 2 database search for sensitive and accurate peptide identification. Molecular \& cellular proteomics : 3 MCP. 2012;11(4):M111 010587.

4 21. Chou MF, Schwartz D. Biological sequence motif discovery using motif-x. Curr Protoc 5 Bioinformatics. 2011; Chapter 13:Unit 13 5-24.

6 22. Petersen B, Petersen TN, Andersen P, Nielsen M, Lundegaard C. A generic method for assignment 7 of reliability scores applied to solvent accessibility predictions. BMC structural biology. 2009;9:51.

8 23. Zhu X, Liu X, Cheng Z, Zhu J, Xu L, Wang F, et al. Quantitative Analysis of Global Proteome and 9 Lysine Acetylome Reveal the Differential Impacts of VPA and SAHA on HL60 Cells. Scientific reports. $10 \quad 2016 ; 6: 19926$.

11 24. Marimuthu A, O'Meally RN, Chaerkady R, Subbannayya Y, Nanjappa V, Kumar P, et al. A 12 comprehensive map of the human urinary proteome. Journal of proteome research. $132011 ; 10(6): 2734-43$. 
1

A

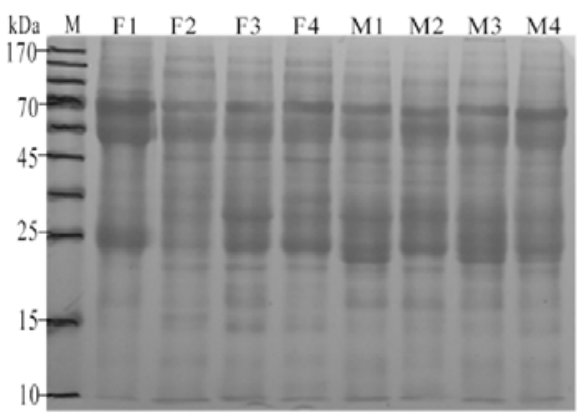

B

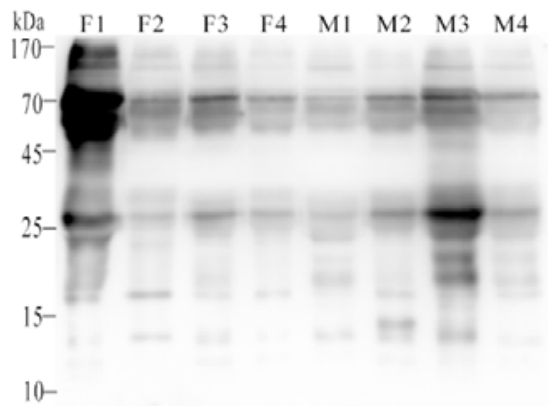

2 Figure 1. Overview of lysine acetylation in urine proteome of healthy humans.

3 (A) SDS-PAGE analysis of urinary proteins followed by transfer to PVDF membrane

4 and staining with Coomassie Brilliant Blue; (B) Western blotting analysis of urinary

5 proteins. (M: male, F: female).

6

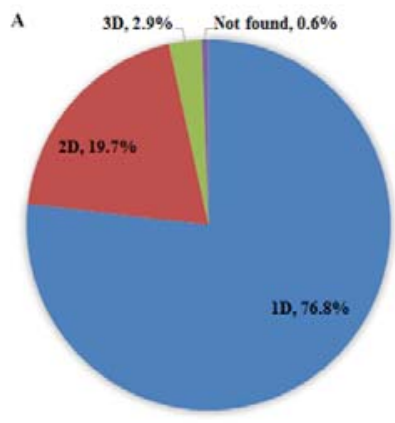

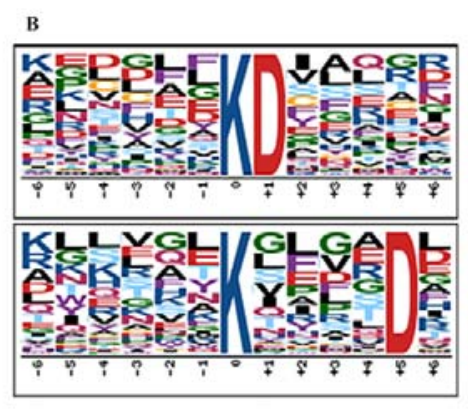

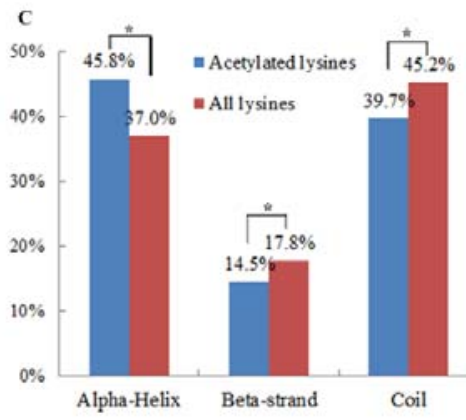

7 Figure 2. Statistics of the lysine-acetylated proteins.

8 (A) Estimation of relative abundance of urinary lysine-acetylated proteins. When the

9315 proteins were searched in the most comprehensive urinary database, 242 were

10 found in the 1D group (proteins detectable when separated by 1D LC) and 71 in the 2D and 3D group (2D: high-pH RPLC coupled LC- MS/MS, 3D: GELFrEE/LP-IEF

12 fraction prior to 2D), which were less abundant proteins. (B) Acetylation motifs and

13 conservation of acetylation sites. (C) Distribution of acetylated and non-acetylated

14 lysine residues in protein secondary structures $(* \mathrm{p}<0.01)$. 
A

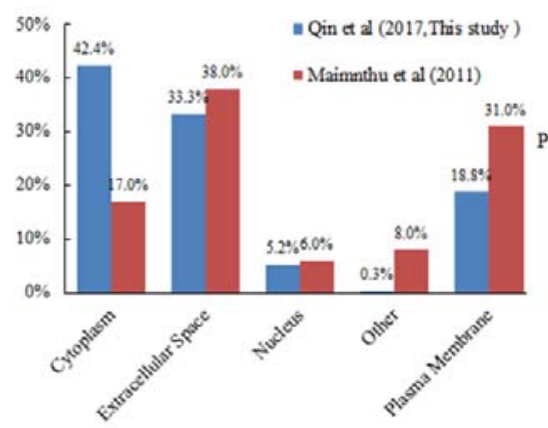

C

Organismal Injury and Abnormalities Endocrine System Disorders

Cell-To-Cell Signaling and Interaction Dermatological Diseases and Conditions Metabolic Disease

Neurological Disease Cell Death and Survival Cellular Growth and Proliferation Free Radical Scavenging Cellular Movement

B
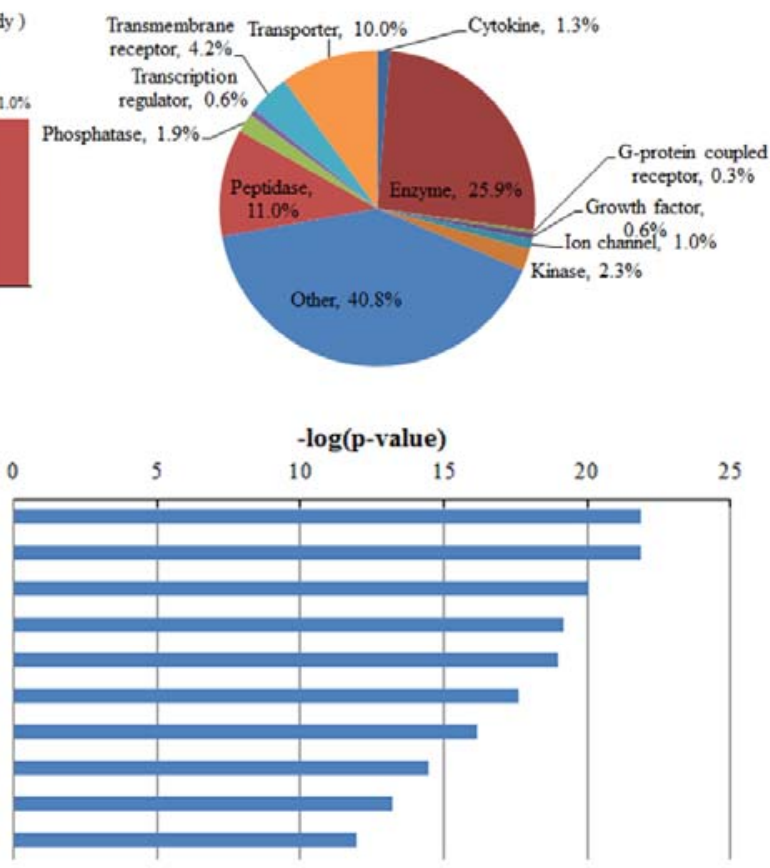

-log(p-value)

D

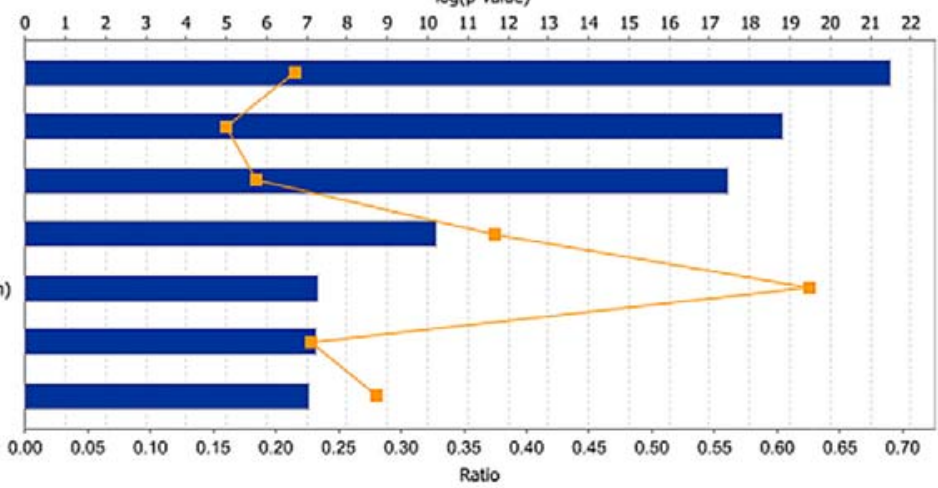

UXR/RXR Activation

Acute Phase Response Signaling

FXR/RXR Activation

Glycolysis 1

Sucrose Degradation V (Mammalian)

Cosgulation System

Gluconeogenesis I
1

2 Figure 3. Functional analysis of the lysine acetylome in urine.

3 The lysine-acetylated proteins identified in this study were classified according to

4 their (A) subcellular location, (B) protein family, (C) enriched bio functions and

5 diseases, and (D) canonical pathways and networks.

\section{Supplementary Material:}

7 The flowing supporting information and the raw MS/MS data have been uploaded to

8 the data repository. (https://figshare.com/s/4983659b9db1d69b7b8c) 
1 Supporting Fig S1. Overview of lysine acetylation in urine proteome of healthy

2 humans. (A) SDS-PAGE analysis of urinary proteins followed by transfer to PVDF

3 membrane and staining with Coomassie Brilliant Blue; (B) Western blotting analysis

4 of urinary proteins. (M: male, F: female).

5 Supporting Table S1. Acetylated peptides (Peptide-Spectrum Matches FDR < $0.1 \%$ ).

6 Supporting Table S2. Acetylated proteins (Unique peptides $\geq 1$ ) with 629 Kac sites

$7 \quad$ (AScore $\geq 59$ ) in normal human urine.

8 Supporting Spectra S1. The represent MS/MS spectra for 761 lysine acetylated

9 peptides.

10 Supporting Spectra S2. The remaining MS/MS spectra for 761 lysine acetylated 11 peptides. 